University of Nebraska - Lincoln

DigitalCommons@University of Nebraska - Lincoln

\title{
Validation of a cell-based ELISA as a screening tool identifyinganti-alphavirus small-molecule inhibitors
}

\author{
Kevin B. Spurgers \\ U.S. Army Medical Research Institute of Infectious Diseases \\ Clarence R. Hurt \\ StatesbProsetta Antiviral, Inc \\ Jeffrey W. Cohen \\ U.S. Army Medical Research Institute of Infectious Diseases \\ Lori T. Eccelston \\ U.S. Army Medical Research Institute of Infectious Diseases \\ Cathleen M. Lind \\ U.S. Army Medical Research Institute of Infectious Diseases \\ See next page for additional authors
}

Follow this and additional works at: https://digitalcommons.unl.edu/usarmyresearch

Spurgers, Kevin B.; Hurt, Clarence R.; Cohen, Jeffrey W.; Eccelston, Lori T.; Lind, Cathleen M.; Lingappa, Vishwanath R.; and Glass, Pamela J., "Validation of a cell-based ELISA as a screening tool identifyingantialphavirus small-molecule inhibitors" (2013). US Army Research. 226.

https://digitalcommons.unl.edu/usarmyresearch/226

This Article is brought to you for free and open access by the U.S. Department of Defense at DigitalCommons@University of Nebraska - Lincoln. It has been accepted for inclusion in US Army Research by an authorized administrator of DigitalCommons@University of Nebraska - Lincoln. 


\section{Authors}

Kevin B. Spurgers, Clarence R. Hurt, Jeffrey W. Cohen, Lori T. Eccelston, Cathleen M. Lind, Vishwanath R. Lingappa, and Pamela J. Glass 


\title{
Validation of a cell-based ELISA as a screening tool identifying anti-alphavirus small-molecule inhibitors
}

\author{
Kevin B. Spurgers ${ }^{a}$, Clarence R. Hurt ${ }^{b}$, Jeffrey W. Cohen ${ }^{a}$, Lori T. Eccelston ${ }^{a}$, \\ Cathleen M. Lind ${ }^{\mathrm{a}}$, Vishwanath R. Lingappa ${ }^{\mathrm{b}}$, Pamela J. Glass ${ }^{\mathrm{a}, *}$ \\ a U.S. Army Medical Research Institute of Infectious Diseases, Frederick, MD 21702, United States \\ b Prosetta Antiviral, Inc., San Francisco, CA 94107, United States
}

\section{Article history:}

Received 21 December 2012

Received in revised form 17 May 2013

Accepted 3 June 2013

Available online $\mathrm{xxx}$

\section{Keywords:}

ELISA

Alphavirus

Small-molecule inhibitor

Screen

Virus yield reduction assay

Venezuelan equine encephalitis virus

(VEEV)

\begin{abstract}
A B S T R A C T
Venezuelan (VEEV), eastern, and western equine encephalitis viruses, members of the genus Alphavirus, are causative agents of debilitative and sometimes fatal encephalitis. Although human cases are rare, these viruses pose a threat to military personnel, and to public health, due to their potential use as bioweapons. Currently, there are no licensed therapeutics for treating alphavirus infections. To address this need, small-molecules with potential anti-alphavirus activity, provided by collaborators, are tested routinely in live alphavirus assays utilizing time-consuming virus yield-reduction assays. To expedite the screening/hit-confirmation process, a cell-based enzyme-linked immunosorbent assay (ELISA) was developed and validated for the measurement of VEEV infection. A signal-to-background ratio of $>900$, and a $z$-factor of $>0.8$ indicated the robustness of this assay. For validation, the cell-based ELISA was compared directly to results from virus yield reduction assays in a single dose screen of 21 compounds. Using stringent criteria for anti-VEEV activity there was $90 \%$ agreement between the two assays (compounds displaying either antiviral activity, or no effect, in both assays). A concurrent compound-induced cell toxicity assay effectively filtered out false-positive hits. The cell-based ELISA also reproduced successfully compound dose-response virus inhibition data observed using the virus yield reduction assay. With available antibodies, this assay can be adapted readily to other viruses of interest to the biodefense community. Additionally, it is cost-effective, rapid, and amenable to automation and scale-up. Therefore, this assay could expedite greatly screening efforts and the identification of effective anti-alphavirus inhibitors.
\end{abstract}

Published by Elsevier B.V.

\section{Introduction}

The majority of viruses within the genus Alphavirus are nonpathogenic to humans. However, select alphaviruses can cause severe disease in humans and represent a significant threat to public health. Venezuelan (VEEV), eastern (EEEV), and western equine

Abbreviations: BSA, bovine serum albumin; CHIKV, Chikungunya virus; CPE, cytopathic effect; EEEV, eastern equine encephalitis virus; ELISA, enzymelinked immunosorbent assay; HRP, horseradish peroxidase; $\mathrm{IC}_{50}, 50 \%$ inhibitory concentration; MAb, monoclonal antibody; MOI, multiplicity of infection; PBS, phosphate-buffered saline; pfu, plaque-forming units; RT, room temperature; S/B, signal to background ratio; $\mathrm{SD}$, standard deviation; $\mathrm{TCID}_{50}$, tissue culture infectious dose 50; TrD, Trinidad donkey strain of VEEV; USAMRIID, United States Army Medical Research Institute of Infectious Disease; VEEV, Venezuelan equine encephalitis virus; WEEV, western equine encephalitis virus.

* Corresponding author at: U.S. Army Medical Research Institute of Infectious Diseases, 1425 Porter Street, Fort Detrick, MD 21702, United States. Tel.: +1 3016194742 .

E-mail addresses: pamela.j.glass.civ@mail.mil, pam.glass@us.army.mil (P.J. Glass). encephalitis (WEEV) viruses, are causative agents of debilitative, acute, and sometimes fatal encephalitis in North, Central, and South America (Strauss and Strauss, 1994). Chikungunya virus (CHIKV), an old world alphavirus, causes chikungunya fever, a febrile illness characterized by severe arthralgia (Schwartz and Albert, 2010; Thiboutot et al., 2010). In 2005, a large outbreak of chikungunya fever began in La Reunion, an island in the Indian Ocean. This outbreak caused over 300,000 cases and 237 deaths (Higgs, 2006), and subsequently spread to India where over 1.5 million cases were recorded. These alphaviruses are naturally maintained in a zoonotic cycle between nonhuman vertebrate hosts and hematophagous mosquito vectors. Natural human cases occur through the bite of an infected mosquito. VEEV, EEEV, and WEEV are additionally recognized as candidates for use as biological weapons, and are classified as category B pathogens by the Centers for Disease Control and Prevention and The National Institutes of Health (Reichert et al., 2009). Given this threat, and the recent reemergence of CHIKV, there is a critical need for an effective, broad-spectrum anti-alphavirus therapeutic. Currently, there are no licensed drugs available for the treatment of VEEV, EEEV, WEEV, or CHIKV infections. 
Alphavirus virions are small, spherical particles $\sim 70 \mathrm{~nm}$ in diameter, with a nucleocapsid core surrounded by a host-derived lipid membrane (Strauss and Strauss, 1994). The nucleocapsid is assembled from 240 copies of viral capsid protein (C). Glycoprotein spikes, composed of trimers of viral E1/E2 heterodimers are embedded in, and extend from the viral membrane. To address the need for alphavirus therapeutics, Prosetta Antiviral, Inc. developed a cell-free, high-throughput screen to identify compounds that interfere with alphavirus capsid assembly (manuscript in preparation). Preliminary hits from this screen, as well as small-molecules from other collaborators, are routinely tested for anti-alphavirus activity using standard, time-consuming, and labor-intensive virus yield-reduction assays (standard plaque assay or tissue culture infectious dose $50\left[\mathrm{TCID}_{50}\right]$ assay). Although these traditional virus titer assays can validate hits from preliminary screens, their use represents a significant bottle-neck in the hit-confirmation process.

The present study describes the development and validation of a cell-based ELISA for the detection and quantitation of VEEV infection in cell monolayers, and its suitability for use in high-throughput screening applications. The ELISA reproduced accurately data from a virus yield reduction assay in a singledose screen of 21 test compounds with potential anti-alphavirus activity, and can also be used in the context of compound dose curve analyses and $\mathrm{IC}_{50}$ (50\% inhibitory concentration) calculations. This assay is significantly less time-, labor-, and material-intensive than virus yield reduction measurements using standard plaque assay or $\mathrm{TCID}_{50}$ assay. A high signal-to-background ratio and high $z$-factor value indicate that the cell-based ELISA method can be adapted successfully for use in a high-throughput scenario. Based on these data, the VEEV cell-based ELISA is suitable for, and will expedite screening efforts to identify anti-VEEV small-molecule inhibitors.

\section{Materials and methods}

\subsection{Virus and cells}

Virus infections were carried out with VEEV (subtype IA/B) Trinidad donkey (TrD) strain obtained from USAMRIID archives. Vero cells were obtained from USAMRIID Virology Department cell culture core lab, and were maintained in Eagle's Minimum Essential Medium (EMEM) supplemented with non-essential amino acids, and $10 \%$ fetal bovine serum (Life Technologies ${ }^{\mathrm{TM}}$, Grand Island, NY). Cells were incubated at $37^{\circ} \mathrm{C}$, in a humidified incubator, with $5 \%$ $\mathrm{CO}_{2}$.

\subsection{Cell-based ELISA}

Confluent Vero cell monolayers in black 96-well plates (Greiner Bio-one, Monroe, NC) were infected with VEEV TrD at various multiplicities of infection (MOIs). MOI calculations utilized a cell number (based on previous cell counts) of $4 \times 10^{4}$ Vero cells per confluent well in a 96-well plate. Virus was diluted in complete medium and was added to cells in $50 \mu \mathrm{l}$ volume. After $1 \mathrm{~h}$ virus was removed and fresh medium added. At indicated time points after infection, cells were fixed in $3.7 \%$ formaldehyde in phosphate-buffered saline (PBS), at $4{ }^{\circ} \mathrm{C}$ for $18-24 \mathrm{~h}$. Cells were washed in PBS and then blocked with $3 \%$ bovine serum albumin (BSA, Sigma-Aldrich, St. Louis, MO) in PBS for $1 \mathrm{~h}$ at room temperature (RT). Anti-VEEV E2 1A4A-1 monoclonal antibody (Roehrig and Mathews, 1985) $(1 \mathrm{mg} / \mathrm{ml}$ ) was diluted in PBS $+3 \%$ BSA and added to cells for $2 \mathrm{~h}$, RT. After washing with PBS, cells were incubated with goat anti-mouse horseradish peroxidase (HRP) secondary antibody (Invitrogen, Grand Island, $\mathrm{NY}$ ) diluted in PBS $+3 \%$ BSA for $1 \mathrm{~h}$, RT. Cells expressing VEEV
E2 protein were detected with Super Signal ELISA Pico chemiluminescence substrate (Thermo Scientific, Waltham, MA) (5 min incubation, RT), and a Spectramax M5 plate reader (Molecular Devices, Sunnyvale, CA).

For the compound screen using the ELISA, compounds were diluted and mixed with VEEV virions for $4 \mathrm{~h}, 37^{\circ} \mathrm{C}$. The compound/virus mixture was then added to Vero cells plated in black 96-well plates. Cells were incubated in the presence virus/compound for $18 \mathrm{~h}$. Cells were then fixed and processed for ELISA as described above. Bafilomycin A1 (Enzo Life Sciences, Farmingdale, NY) was used as a positive control VEEV-inhibitory compound in the ELISA (Hunt et al., 2011).

\subsection{Cell viability}

Compounds were diluted to the indicated concentrations in complete media and added to Vero cells in black 96-well plates, in $100 \mu \mathrm{l}$ final volume. Cells were incubated for $48 \mathrm{~h}$, at $37^{\circ} \mathrm{C}$, and then moved to RT for $30 \mathrm{~min}$. Cell Titer Blue reagent ( $20 \mu \mathrm{l}$, Promega, Madison, WI) was added to cell culture supernatant in each well, and incubated for $3 \mathrm{~h}$ at $37^{\circ} \mathrm{C}$. Fluorescence was then measured in each well with a Spectramax M5 plate reader.

\subsection{Virus yield reduction assay}

Vero cells in 24-well plates were infected with VEEV TrD (MOI 10 ), for $1 \mathrm{~h}, 37^{\circ} \mathrm{C}$, in $100 \mu \mathrm{l}$ volume. After infection, the virus inocula were removed, cells were washed twice with serum-free medium. Complete medium ( $2 \% \mathrm{FBS}+$ penicillin $0.5 \mathrm{mg} / \mathrm{ml}$, streptomycin $0.5 \mathrm{mg} / \mathrm{ml}$ ) containing compound was then added to the cells (triplicate wells for each compound/concentration), and incubated for $24 \mathrm{~h}, 37^{\circ} \mathrm{C}$. Cell culture supernatants from each well of the 24-well plate were collected. Virus yield was determined by $\mathrm{TCID}_{50}$ assay. Briefly, supernatants were diluted in 96 -well plates (five replicate dilution series for each well of the 24-well plate). A 10 -fold dilution series was generated from $10^{-1}$ to $10^{-8}$. The serial dilutions were then transferred to Vero cells in 96-well plates, and incubated for $6 \mathrm{~d}$. Medium was removed and cells were fixed and stained with $0.05 \%$ crystal violet in $3.7 \%$ formaldehyde for $30 \mathrm{~min}$ at RT. Stain was removed and cells were rinsed with water and air dried. Cytopathic effect (CPE) was observed by visual inspection. The two virus dilutions where CPE transitioned from $>50 \%$ (three or more replicate wells with $\mathrm{CPE}$ ) to $<50 \%$ of replicates with CPE were recorded. $\mathrm{TCID}_{50}$ was determined by calculations described previously (Reed and Muench, 1938).

\subsection{Statistics}

For replicate 96-well plates containing cells infected with VEEV (positive controls) or not infected (negative controls), $t$ tests were used to compare plate 1 to plate 2 on each of two days to assess plate-to-plate differences. For replicate 96-well plates containing cells infected with VEEV or not infected, $t$-tests were used to compare plates from day 1 to plates from day 2 to assess day-to-day differences. For 96-well plates containing cells infected with VEEV in the presence of a cohort of 21 potential anti-VEEV compounds, the correlation coefficient $(r)$ between the virus yield reduction assay and the cell-based ELISA was calculated, and used to determine if the correlation was statistically significant. Spearman's rank correlation was utilized. Using the cell-based ELISA, and eight-point dose curve was analyzed for compound 22 . The $\mathrm{IC}_{50}$ value was calculated using probit analysis. 


\section{Results}

\subsection{Optimization of a VEEV cell-based ELISA and characterization of infection kinetics}

In previous experiments, monoclonal antibody (MAb) 1A4A-1, directed against VEEV E2 protein, has proven effective at detecting viral antigen in immunofluorescence assays (Parker et al., 2010). This antibody was evaluated for performance in a cell-based ELISA format for the detection and quantitation of VEEV-infected Vero cell monolayers in 96-well plates. MAb 1A4A and HRP-conjugated secondary antibodies were titrated to determine optimal concentrations yielding maximized signal/background ratios (S/B). Vero cells were infected with VEEV, at an MOI of 0.01 plaque-forming units per cell ( $\mathrm{pfu} / \mathrm{cell}$ ), and incubated for $18 \mathrm{~h}$. Cells were then fixed and processed for cell-based ELISA. Four dilutions of MAb 1A4A were tested with four dilutions of HRP-conjugated secondary antibody. After addition of the HRP substrate, luminescent signal was recorded with a plate reader. A primary antibody dilution of 1:4000, with a secondary antibody dilution of either $1: 5000$ or $1: 10,000$ yielded $\mathrm{S} / \mathrm{B}$ ratios $>900$. All subsequent experiments were carried out using a 1:4000 dilution of $1 \mathrm{~A} 4 \mathrm{~A}$, and HRP-conjugated secondary antibody diluted 1:10,000.

Experiments were performed to determine the appropriate virus dose (MOI) and infection time point to be used in smallmolecule screening efforts. Optimal parameters are necessary for the detection of inhibition of infection (virus spread) in the presence of compounds with anti-VEEV activity. These parameters are especially relevant when screening for inhibitors of viruses with extremely fast replication cycles, such as VEEV (4h) (Strauss and Strauss, 1994). Vero cells were infected with VEEV at MOIs of 0 , $0.0001,0.001,0.01,0.1$, and $1 \mathrm{pfu} /$ cell. Infected cells were incubated for 6,18 , or $24 \mathrm{~h}$ before fixation and processing for cell-based ELISA (Fig. 1A). A low level of infection was detected at $6 \mathrm{~h}$ using MOIs of 1 and 0.1 . In contrast, the luminescent signal was near maximum for most MOIs tested $24 \mathrm{~h}$ after infection. When measured at $18 \mathrm{~h}$ after infection, the ELISA signal was virus dose-dependent, and did not reach maximum levels (compared to $24 \mathrm{~h}$ data). High variability was consistently seen at the very low MOI of 0.0001 , as some replicate wells had infected cells and others no infection was detected. Based on the $18 \mathrm{~h}$ virus dose-response curve, subsequent small-molecule screening experiments were performed using MOI 0.01 , infected for $18 \mathrm{~h}$.

The suitability of the ELISA as an assay for high-throughput screening was investigated next. Cells in two replicate 96-well plates were infected with VEEV (MOI $0.01,18 \mathrm{~h}$ ), or not infected, on two separate days (four plates total in analysis). Cells were fixed and processed for ELISA (Fig. 1B). T-tests were utilized to assess plate-to-plate and day-to-day variability. Briefly, $t$-tests compared ELISA luminescence signal between plate 1 and 2 for each day (experiment 1 and 2) for negative controls and positive controls separately and combined. Likewise $t$-tests compared luminescence signal between plates of experiments 1 and experiment 2 . In each case, the resulting $p$-values indicate that no statistically significant differences were found (not shown). This analysis demonstrated low plate-to-plate and day-to-day variability for the VEEV cellbased ELISA. Using averaged data from all four plates, a $z$-factor of 0.82 was calculated for this assay (Table 1 ).

\subsection{Comparison of cell-based ELISA to virus yield reduction assay in a limited screen for potential anti-VEEV small-molecule inhibitors}

A cohort of potential anti-VEEV compounds was supplied to USAMRIID by Prosetta Antiviral, Inc. These compounds were subsequently tested for anti-VEEV activity using a virus yield reduction

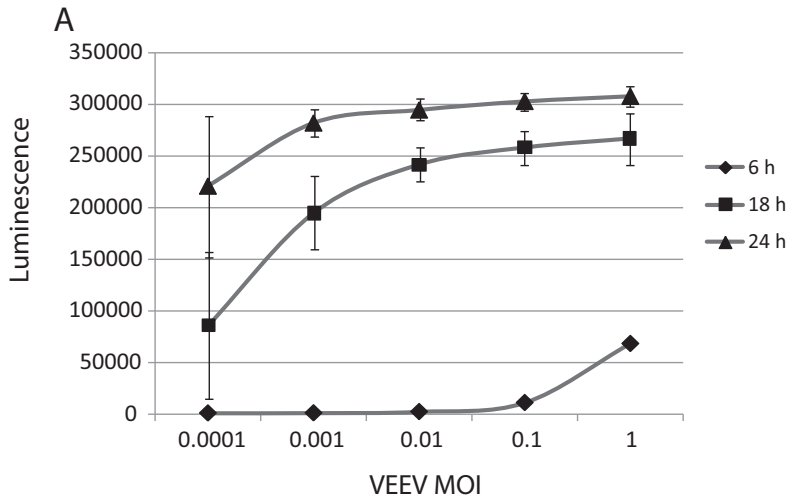

B

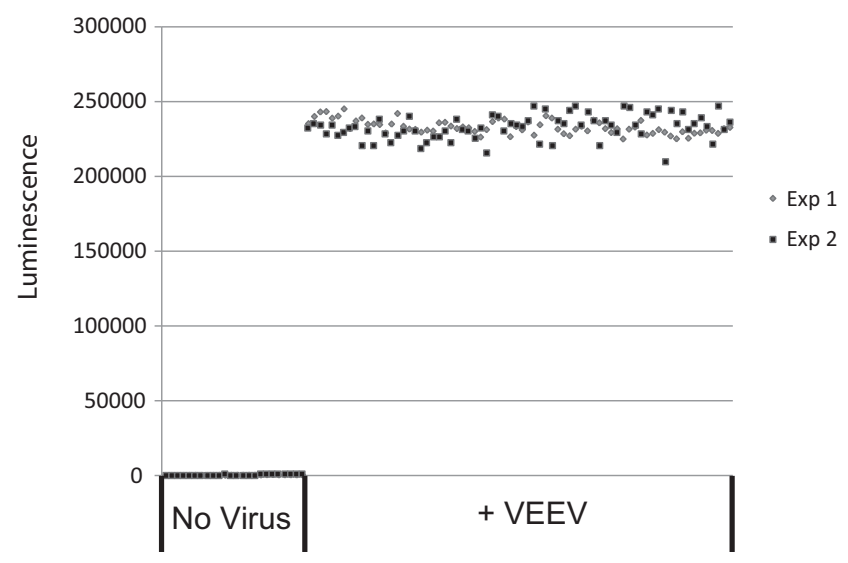

Fig. 1. Evaluation and optimization of a cell-based ELISA for the quantitation of VEEV infection in 96-well plates. (A) A virus dose response and time course experiment was performed to examine the kinetics of VEEV infection as measured by the cell-based ELISA. Vero cells were infected at the indicated MOIs for 6, 18, or $24 \mathrm{~h}$ Luminescent ELISA signal is plotted for each MOI and time point. (B) Determination of plate-to-plate and day-to-day variability of cell-based ELISA data. Duplicate plates were infected with VEEV MOI 0.01 for $18 \mathrm{~h}$, or not infected, and then processed for ELISA. Two independent experiments (Exp 1 and Exp 2) were carried out on separate days. For each experiment, luminescence data for each well on duplicate plates were averaged and plotted.

assay and the cell-based ELISA. For virus yield reduction assay, cells were infected with VEEV (MOI 10) for $1 \mathrm{~h}$. After incubation, excess virus was removed, cells were washed with serum-free medium, and complete medium containing compound $(10 \mu \mathrm{M})$, or vehicle control (dimethyl sulfoxide, DMSO, final concentration $0.1 \%$ ) was added. Infected cells were incubated with compound for $24 \mathrm{~h}$. The cell culture supernatant was then collected and VEEV titer measured by $\mathrm{TCID}_{50}$ assay (Fig. $2 \mathrm{~A}$ ). Several compounds reduced VEEV virus yield by $>4$ logs. Compounds with intermediate anti-VEEV activity, or no activity, were also detected (Fig. 2A and Table 2). Previous experiments demonstrated that DMSO does not influence VEEV titer in this assay (not shown). This same cohort of compounds was also tested for anti-VEEV activity using the cell-based ELISA. A screening protocol was established that would detect effectively compounds that act directly on virions, or that act on intracellular protein targets. To this end, VEEV virions were mixed

Table 1

Z-factor determination for VEEV cell-based ELISA.

\begin{tabular}{lrlll}
\hline Wells & \multicolumn{1}{c}{$n$} & Mean $^{\mathrm{a}}$ & SD & $Z^{\prime}$ \\
\hline Non-infected & 96 & 2.65 & 0.14 & 0.82 \\
VEEV-infected & 288 & 5.37 & 0.02 & \\
\hline
\end{tabular}

SD, standard deviation.

${ }^{\text {a }} \log _{10}$-transformed luminescence values. 

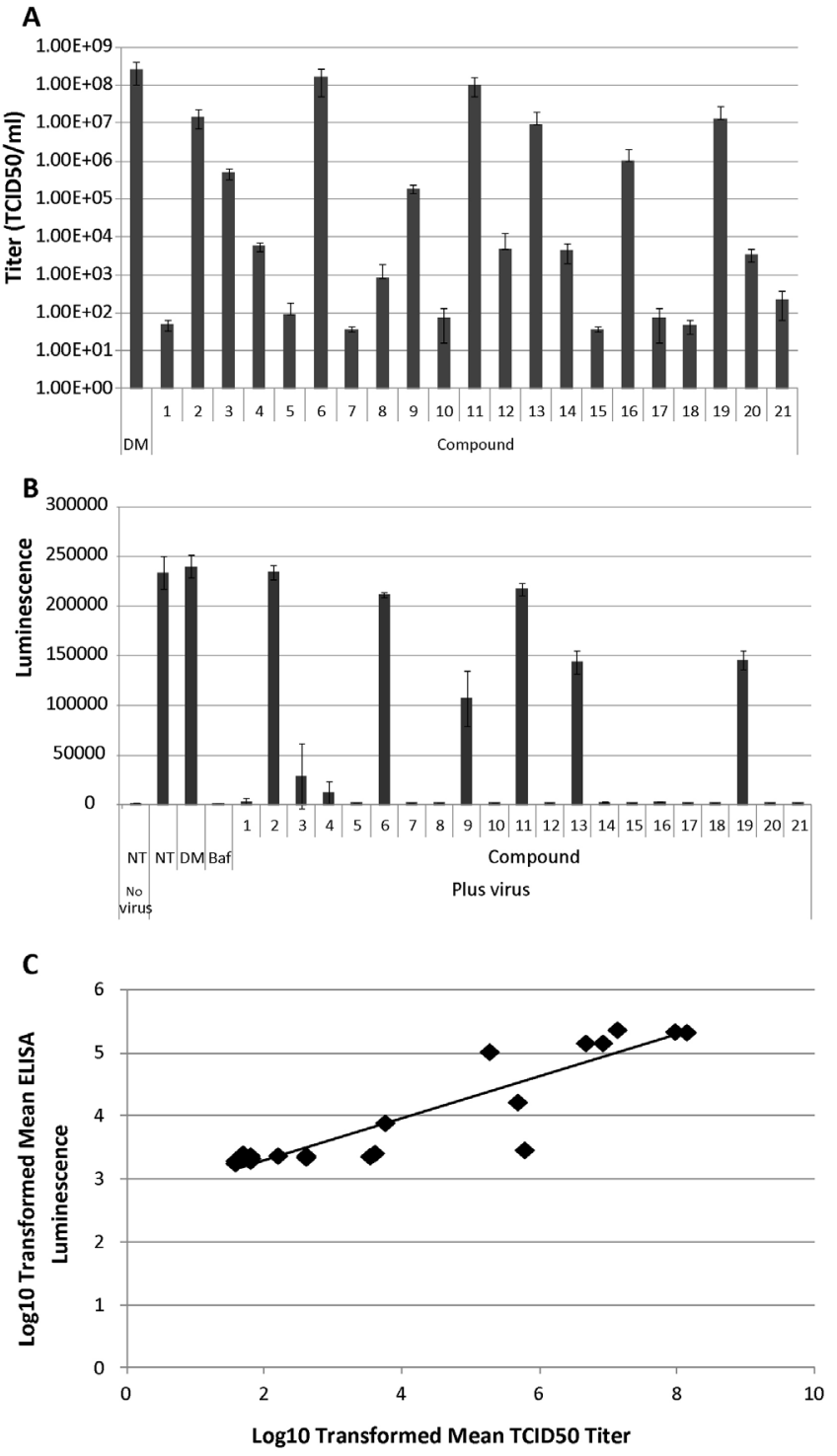

D

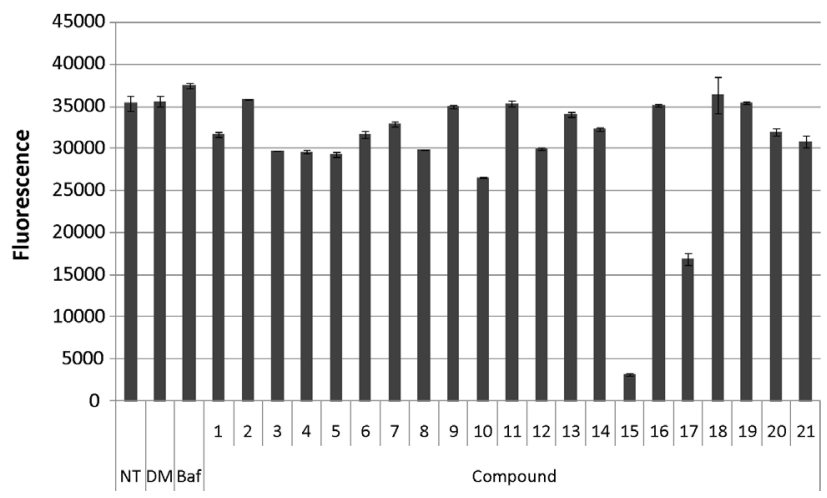

Fig. 2. Comparison of virus yield reduction assay and cell-based ELISA in a small screen to identify anti-VEEV compounds. (A) Twenty-one small-molecule compounds were screened for the ability to reduce VEEV yield. Titers in cell culture supernatants were determined by $\mathrm{TCID}_{50}$ assay. Cells were infected with VEEV in 24-well plates for $1 \mathrm{~h}$. After the infection period, medium was added containing compound $(10 \mu \mathrm{M})$, or DMSO as a control (DM). VEEV titer $\left(\mathrm{TCID}_{50} / \mathrm{ml}\right)$ was measured in cell culture supernatants $24 \mathrm{~h}$ after the start of infection, and was plotted for each compound. (B) The same group of compounds was examined for anti-VEEV activity using the cell-based ELISA. Virions were pre-treated with compound, or not treated (NT) and subsequently used to infect cells. The compound + virus mixture remained on cells through the $18 \mathrm{~h}$ incubation period. DMSO (DM) had no effect
Table 2

Results of small-molecule screen. Correlation between ELISA and virus yield reduction assay data.

\begin{tabular}{rll}
\hline Compound & ELISA (\% inhibition) & TCID50 (decrease in titer) \\
\hline 1 & $>80 \%$ & $>4 \log$ \\
2 & $<20 \%$ & $\sim 1 \log$ \\
3 & $>80 \%$ & $>2 \log$ \\
4 & $>80 \%$ & $>4 \log$ \\
5 & $>80 \%$ & $>4 \log$ \\
6 & $<20 \%$ & No decrease \\
7 & $>80 \%$ & $>4 \log$ \\
8 & $>80 \%$ & $>4 \log$ \\
9 & $>50 \%$ & $\sim 3 \log$ \\
10 & $>80 \%$ & $>4 \log$ \\
11 & $<20 \%$ & No decrease \\
12 & $>80 \%$ & $>4 \log$ \\
13 & $<50 \%$ & $<2 \log$ \\
14 & $>80 \%$ & $>4 \log$ \\
15 & $>80 \%$ & $>4 \log$ \\
16 & $>80 \%$ & $>2 \log$ \\
17 & $>80 \%$ & $>4 \log$ \\
18 & $>80 \%$ & $>4 \log$ \\
19 & $<50 \%$ & $<2 \log$ \\
20 & $>80 \%$ & $>4 \log$ \\
21 & $>80 \%$ & $>4 \log$ \\
\hline
\end{tabular}

with individual compounds and incubated at $37^{\circ} \mathrm{C}$ for $4 \mathrm{~h}$. The virion/compound mixture ( $10 \mu \mathrm{M}$ compound final concentration) was then added to Vero cell monolayers in a 96-well plate. This infection mixture remained on the cells for $18 \mathrm{~h}$. Cells were then fixed and processed for ELISA. The data obtained with this assay closely mirrored those of the virus yield reduction assay (Fig. 2B and Table 2). Of the 21 compounds tested, 15 compounds reduced ELISA signal by $>80 \%$ of control (infected cells without compound treatment). Each of these hits reduced virus yield by $>2$ logs, with most $(13 / 15)$ showing a $>4 \log$ reduction in virus titer. Spearman's rank correlation was utilized to evaluate the relationship between $\mathrm{TCID}_{50}$ titers and ELISA luminescence for the cohort of potential anti-VEEV compounds. The resulting correlation coefficient was $r=0.88$, and the corresponding $p$-value was $p<0.0001$, indicating that there is evidence of a linear relationship between the virus yield reduction and ELISA results (Fig. 2C). Overall, the cell-based ELISA for quantitation of VEEV infection accurately recapitulated virus yield reduction assay titer measurements made with $\mathrm{TCID}_{50}$ assay.

The observed compound-dependent reduction in ELISA signal, or reduction in virus yield, could be the result of potent VEEV inhibition, or the result of compound-induced cell toxicity. Compounds producing significant toxicity could present as false-positives in either of these assays. To help identify and filter out false positives, at the time of infection for the ELISA, cells in a replicate plate were exposed to compound without virus infection. Cells were incubated for $48 \mathrm{~h}$ at $37^{\circ} \mathrm{C}$. Cell viability was then measured using Cell Titer Blue assay (Fig. 2D). Most compounds did not produce significant toxicity, as measured by this particular assay. Compound 15 was clearly toxic and reduced the viability-dependent fluorescent signal to $9 \%$ of the control wells. These data suggest that compound 15 is a false-positive hit in the ELISA and virus yield reduction assays, and would be filtered out of further analysis. Two other compounds

on VEEV infection-dependent ELISA signal. Bafilomycin (Baf, $1 \mu \mathrm{M}$ ) was used as a positive control and effectively inhibited VEEV infection. Several compounds dramatically reduced ELISA signal after VEEV infection. (C) Average ELISA signal vs. average $\mathrm{TCID}_{50}$ titer for each of 21 compounds. For each compound, ELISA luminescence data and TCID 50 titer data were $\log _{10}$ transformed and plotted on a scatter plot. The linear regression line is shown. (D) Cell toxicity assay. Vero cells were treated with each compound $(10 \mu \mathrm{M})$, DMSO, or bafilomycin $(1 \mu \mathrm{M})$ for $48 \mathrm{~h}$. Cell viability was then measured using Cell Titer Blue assay. Viability-dependent fluorescent signal was recorded and plotted for each treatment. Most compounds had little effect on cell viability as measured by this assay. 
A

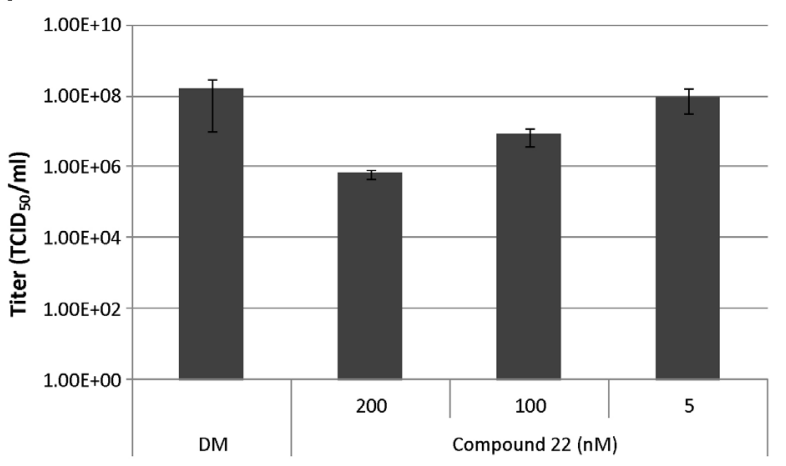

B

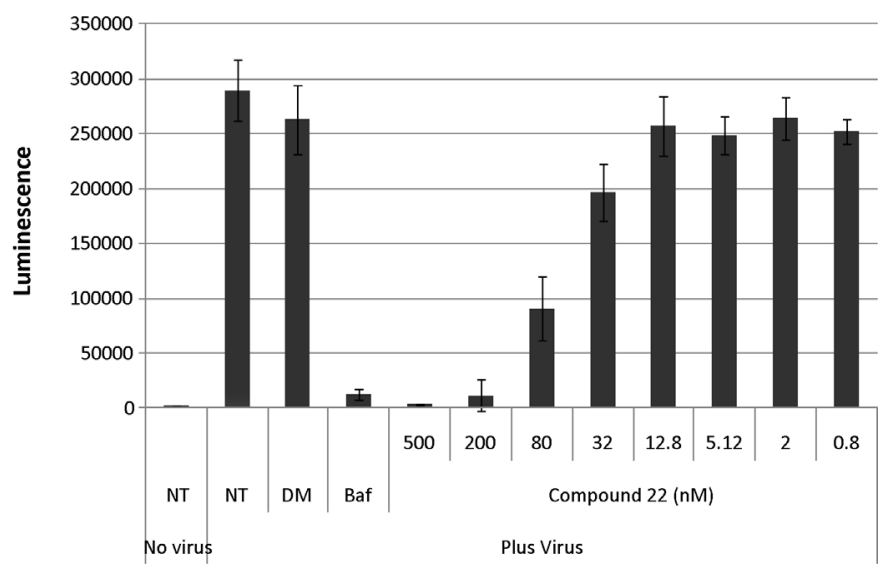

C

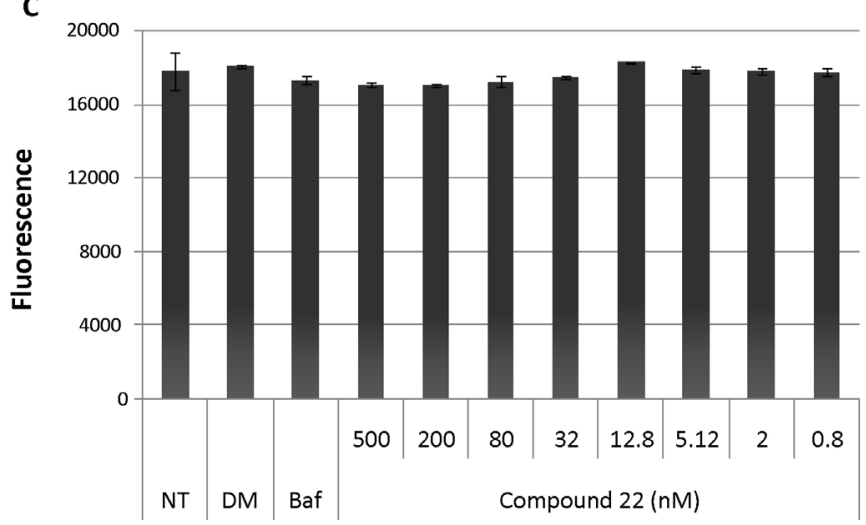

Fig. 3. Comparison of virus yield reduction assay and cell-based ELISA in a dose response analysis of a non-toxic compound with anti-VEEV activity. (A) Compound 22 was tested at three concentrations for the ability to decrease VEEV yield. A dosedependent inhibition of VEEV, compared to DMSO control (DM), was observed. (B) An 8-point dose response for compound 22 was tested for VEEV inhibition with the cell-based ELISA. DMSO (DM) had little or no effect, while bafilomycin (Baf) significantly inhibited VEEV. Treatment of cells with compound 22 resulted in a dose-dependent inhibition of VEEV infection. (C) Cell Titer Blue viability assay after $48 \mathrm{~h}$ treatment with several doses of compound 22 . No compound toxicity was detected with this assay.

(10 and 17 ) produced a viability signal $<80 \%$ of control cells, and should be considered potential false-positives.

\subsection{Dose-dependent anti-VEEV activity of compound 22 measured with virus yield reduction assay and ELISA}

The VEEV-specific cell-based ELISA effectively detected smallmolecule compounds with anti-VEEV activity in a single dose screen. These data also suggested that the ELISA could detect compounds with intermediate or modest antiviral activity. The performance of this assay was examined in the context of a compound dose-response experiment. Compound 22 demonstrated a dosedependent inhibition of VEEV production in Vero cells as measured by the virus yield reduction assay (Fig. 3A). Treatment of cells with $200 \mathrm{nM}$ compound 22 produced an $\sim 2 \log$ reduction in VEEV titer $24 \mathrm{~h}$ after infection compared to DMSO-treated control cells. This virus inhibition was lost at a compound concentration of $5 \mathrm{nM}$. Using the cell-based ELISA and compound 22, an 8-point dose curve analysis was performed (Fig. 3B). Starting with $500 \mathrm{nM}$, a 2.5-fold compound dilution series was generated and tested. Cells were treated with compound and processed for ELISA as described for the single-dose screen. Bafilomycin A1 was used as a control and effectively inhibited VEEV infection in this assay. The cell-based ELISA method detected dose-dependent anti-VEEV activity of compound 22. Using DMSO as the positive control, an $\mathrm{IC}_{50}$ value of $50 \mathrm{nM}$ was calculated for this compound. The same compound dilutions were added to cells, without virus infection, and incubated for $48 \mathrm{~h}$. At this time point no cellular toxicity was detected with this assay at any compound concentration tested (Fig. 3C).

\section{Discussion}

Screening assays to identify small-molecules with antiviral activity can take several forms, each with their own advantages and disadvantages. On one end of the spectrum are in vitro cell-free biochemical screens identifying inhibitors of a known and highly purified protein target with a defined activity. Knowing the protein target of identified inhibitors greatly facilitates downstream drugdevelopment efforts. However, the narrow focus of these assays, searching for inhibitors of one specific protein, can also be described as a disadvantage. Information regarding cell permeability or toxicity is also not readily available. Biochemical screens are typically adapted easily to automation in 384- or 1536-well plate format, achieving true high-throughput status, and do not require biosafety level-3 (BSL-3) or BSL-4 high containment laboratories and the restrictions that go along with working in that environment. This study reports a cell-based screening strategy on the other end of the spectrum, directly identifying inhibitors of authentic, pathogenic VEEV.

In the cell-based ELISA screen described herein, the target of any effective inhibitors is not known. However, this experimental design allows investigators to cast a wide net, potentially identifying compounds that act directly on viral proteins, or on host proteins. Additionally, compounds acting at any stage of the viral lifecycle, from initial attachment to release of nascent viral particles can be identified. In this assay, effective compounds, by definition, have access to cellular and viral proteins. Cell toxicity data can be obtained easily by running viability assays on duplicate plates, but without virus infection. This strategy does require BSL3 containment. While it is possible to carry out initial large screens in BSL1 or BLS2 laboratories using alternative viral reagents, such as pseudotyped virus, attenuated viruses (i.e. VEEV vaccine strain TC83), or non-pathogenic viral replicon particles (VRPs), hits must ultimately be tested against authentic virus. By screening directly against wild-type pathogenic VEEV, the lead-hit generation process is expedited greatly.

A possible disadvantage to a cell-based ELISA screening format is the requirement of a suitable antibody detecting viral antigen. These reagents are not readily available for some viruses. The ELISA format is also more labor intensive than other possible screening strategies for alphavirus inhibitors. For example, most alphavirus infections produce a pronounced cytophathic effect (CPE) in infected cells. This virus-induced loss of cell viability, or 
prevention of cell death by effective small-molecule inhibitors, can be measured easily by common cell viability assays. Many of these assays require the addition of only a single reagent to the cell culture, a short incubation time before reading the assay, and are performed easily in 96- or 384-well plates. However, these assays produced much lower signal-to-background ratios and $z$-factors compared to the cell-based ELISA. The detection of a significant loss of cell viability has also required either high MOIs or long post-infection incubation times before reading the assay $(72-96 \mathrm{~h}$, data not shown). Compounds showing significant but not complete inhibition of VEEV infection were detected more easily with the cell-based ELISA compared to several viability assays tested (not shown).

A small number of screens utilizing diverse methodologies to identify inhibitors of alphavirus infection have been reported. For example, in vitro cell-free biochemical screens can be employed to identify inhibitors of purified viral proteins (when protein activity is known and amenable to assay development). This approach has been applied to Semliki Forest virus (SFV) nsP1 protein (Lampio et al., 1999). SFV nsP1 has methyltransferase activity, and is involved in the capping of viral RNAs. Lampio et al. screened 58 cap analogs for the ability to inhibit nsP1 methytransferase activity. Several inhibitors were identified with Ki values $40-400 \mu \mathrm{M}$. The authors did not report cell toxicity testing or activity against live virus in cell culture. Another cell-free approach, possible when viral protein structural information is available, is in silico compound screening. Using molecular modeling, compounds were designed and synthesized that target the hydrophobic binding pocket of SINV capsid protein (Kim et al., 2005). Select compounds inhibited virus production effectively and were not toxic to uninfected BHK cells. Cell-based assays reported in the literature include WEEV replicons and SINV expressing luciferase as a marker protein (Peng et al., 2009; Pohjala et al., 2008).

Although screens for anti-alphavirus inhibitors can take several forms, identified compounds must be tested ultimately for activity against authentic, wild-type, pathogenic virus in cell culture experiments. Although plaque assay remains a gold standard in the eyes of many investigators, this technique is time-consuming, labor intensive, and is not well suited for testing a large number of compounds, and generating detailed dose response curves. The VEEV cell-based ELISA described here does not rely on the use of nonpathogenic alphaviruses, reporter systems, recombinant viruses, or replicons. Additionally, the assay does not require overly expensive or unwieldy equipment that is difficult to introduce, maintain, and use in a high-containment laboratory setting. With available antibodies, this assay can be adapted readily to other viruses of interest to the biodefense community. Antibodies detecting EEEV and WEEV glycoproteins for use in this ELISA assay format have also been validated. Additionally, the assay is cost effective, rapid, and amenable to automation and scale-up. Therefore, this assay could expedite screening efforts and the identification of effective anti-alphavirus inhibitors greatly.

\section{Acknowledgements}

The authors would like to thank Diana Fisher for her scientific input and statistical analysis, as well as Elaine Morazzani and Victor Rivera for critical review of the paper. Opinions, interpretations, conclusions, and recommendations are those of the authors and are not necessarily endorsed by the U.S. Army. During a portion of this research, KBS was appointed to the Postgraduate Research Participation Program administered by the Oak Ridge Institute for Science and Education (ORISE) through an interagency agreement between the U.S. Department of Energy and USAMRMC. VRL is founder, Chief Technology Officer and Co-CEO of Prosetta Antiviral, Inc. This work was supported in part by the Defense Threat Reduction Agency (DTRA); project number CBM.THRV.01.10.RD.017.

\section{References}

Higgs, S., 2006. The 2005-2006 Chikungunya epidemic in the Indian Ocean. Vector Borne Zoonotic Dis 6, 115-116.

Hunt, S.R., Hernandez, R., Brown, D.T., 2011. Role of the vacuolar-ATPase in Sindbis virus infection. Journal of Virology 85, 1257-1266.

Kim, H.Y., Patkar, C., Warrier, R., Kuhn, R., Cushman, M., 2005. Design, synthesis, and evaluation of dioxane-based antiviral agents targeted against the Sindbis virus capsid protein. Bioorganic and Medicinal Chemistry Letters 15, 3207-3211.

Lampio, A., Ahola, T., Darzynkiewicz, E., Stepinski, J., Jankowska-Anyszka, M., Kaariainen, L., 1999. Guanosine nucleotide analogs as inhibitors of alphavirus mRNA capping enzyme. Antiviral Research 42, 35-46.

Parker, M.D., Buckley, M.J., Melanson, V.R., Glass, P.J., Norwood, D., Hart, M.K., 2010. Antibody to the E3 glycoprotein protects mice against lethal venezuelan equine encephalitis virus infection. Journal of Virology 84, 12683-12690.

Peng, W., Peltier, D.C., Larsen, M.J., Kirchhoff, P.D., Larsen, S.D., Neubig, R.R., Miller, D.J., 2009. Identification of thieno[3,2-b]pyrrole derivatives as novel small molecule inhibitors of neurotropic alphaviruses. Journal of Infectious Diseases 199, 950-957.

Pohjala, L., Barai, V., Azhayev, A., Lapinjoki, S., Ahola, T., 2008. A luciferase-based screening method for inhibitors of alphavirus replication applied to nucleoside analogues. Antiviral Research 78, 215-222.

Reed, L.J., Muench, H., 1938. A simple method of estimating fifty percent endpoints. American Journal of Hygiene 27, 493-497.

Reichert, E., Clase, A., Bacetty, A., Larsen, J., 2009. Alphavirus antiviral drug development: scientific gap analysis and prospective research areas. Biosecurity and Bioterrorism 7, 413-427.

Roehrig, J.T., Mathews, J.H., 1985. The neutralization site on the E2 glycoprotein of Venezuelan equine encephalomyelitis (TC-83) virus is composed of multiple conformationally stable epitopes. Virology 142, 347-356.

Schwartz, O., Albert, M.L., 2010. Biology and pathogenesis of chikungunya virus. Nature Reviews Microbiology 8, 491-500.

Strauss, J.H., Strauss, E.G., 1994. The alphaviruses: gene expression, replication, and evolution. Microbiological Reviews 58, 491-562.

Thiboutot, M.M., Kannan, S., Kawalekar, O.U., Shedlock, D.J., Khan, A.S., Sarangan, G., Srikanth, P., Weiner, D.B., Muthumani, K., 2010. Chikungunya: a potentially emerging epidemic? PLOS Neglected Tropical Diseases 4, e623. 